\title{
Effective Means of Teaching and Developing Emotional Intelligence in the Corrections Industry
}

\author{
Richard Skiba \\ LRES Training Management, Melbourne, Australia \\ Email: richard@skiba.com.au
}

How to cite this paper: Skiba, R. (2020). Effective Means of Teaching and Developing Emotional Intelligence in the Corrections Industry. Advances in Applied Sociology, 10, 187-199.

https://doi.org/10.4236/aasoci.2020.106012

Received: May 13, 2020

Accepted: June 5, 2020

Published: June 8, 2020

Copyright ( 2020 by author(s) and Scientific Research Publishing Inc. This work is licensed under the Creative Commons Attribution International License (CC BY 4.0).

http://creativecommons.org/licenses/by/4.0/

\begin{abstract}
In a recent skills forecast undertaken by Australian Industry Standards (Australian Industry Standards, 2019) related to the Corrections Industry, the Industry Reference Committee, through its consultations, determined that Correctional Officers need to interact with a diverse and varied range of people at any given time and they may also encounter high-tension situations. Given the nature of the Corrections industry, soft skills gain more prominence in this space. One of the primary soft skills identified is emotional intelligence. This paper firstly contextualises the need for development of emotional intelligence in Correctional Officers such that this skill can be effectively utilised in a rehabilitative environment. The paper recognises that an ability to read, interpret, comprehend, and react to inmate emotions as well as to manage their own emotions can impact on the Correctional Officer's work environment and well-being, as well as their safety, and also positively impacts on the inmates. Increased emotional intelligence may contribute to the prevention of security incidents in a corrections facility. The CSC Correctional Services Training Package does not include units of competency specific to defining a competency standard for utilisation of emotional intelligence in a corrections setting. The majority of the training in this area is conducted internally by institutions, rather than by Registered Training Organisations, and as such, a range of performance criteria that can be used for program definition is outlined such that adult learning principles can be integrated into the delivered programs. Likewise, a suitable delivery model, using Learning Conferences, is specified as a potential delivery model.
\end{abstract}

\section{Keywords}

Emotional Intelligence, Corrections, Vocational Education, Adult Learning 


\section{Introduction}

Australian Industry Standards (2019) note that the industry is moving towards a more rehabilitative model rather than an institutional one, aiming to improve offenders' physical and mental welfare. There will also be benefits to the wellbeing of Correctional Officers. Application of emotional intelligence is a core skill required in a rehabilitative model.

Salovey and Mayer (1990), as cited by Basu (2013), created the term "Emotional Intelligence". Salovey and Mayer describe it as "a form of social intelligence that involves the ability to monitor one's own and others feelings and emotions, to discriminate among them and to use this information to guide one's thinking and action". Pittaro (2020) informs that emotional intelligence is loosely defined as the capacity or ability to be aware of, control, including an expression of one's emotions. This extends, likewise, as an ability or capacity to cope with interpersonal relationships judiciously and empathetically. Pittaro elaborates that this is essentially, self-regulation of an individual's own emotions and being able to "read" the body cues and emotions of others. Gaining emotional intelligence, and its use, can give a person self-control under stressful situations, such as being experienced in working in corrections, and the ability to remain calm while assessing the situation, again, particularly important in a corrections setting. Emotional intelligence is a critical skill for correctional officer dealing with inmates, where these regular interactions can be both challenging and draining (Pittaro, 2017a).

A more recent model of emotional intelligence is that presented by Goleman in 2012 (Ifelebuegu, Martins, Theophilus, \& Arewa, 2019). This model presents four principal domains and nineteen confidences. The four domains comprise self-awareness, social awareness, self-management and relationship management.

Emotional intelligence influences how we cope with disappointment, frustration, anger, and a range of other emotions by learning how to control these emotions. In corrections, this is 100 percent critical. An individual losing their temper could easily lead to an incident in which a correctional officer uses excessive force or worse yet, deadly force (Pittaro, 2020). Utilising emotional intelligence can help a Correctional Officer to "quickly, effectively and professionally assess each situation and act appropriately" (Pittaro, 2020). Australian Industry Standards (2019) concur, outlining that the Corrections workforce needs to rely on individual cognitive skills and emotional intelligence to build rapport and sympathy with offenders in order to communicate effectively and resolve or de-escalate conflicts. The World Economic Forum (2018), cited in Australian Industry Standards (2019), has also indicated that skills such as critical thinking, leadership, and emotional intelligence will be in demand in the next four years.

\section{Discussion}

\subsection{Emotional Intelligence in Corrections}

The Queensland Government (2020) specifies that Custodial Correctional Offic- 
ers should possess a number of attributes to undertake the role. These include self-confidence and an ability to remain calm under pressure, implement effective conflict management and problem-solving skills, initiative and the ability to "think on your feet", ability and willingness to follow rules, policies and procedures, professionalism and integrity, and empathy, emotional intelligence and cultural awareness.

At the core of emotional intelligence is the ability to monitor our own emotions as well as the emotions of others (Pittaro, 2020). This requires the four essential skills outlined by Golman in the emotional intelligence model. These four skills are further divided into two categories, namely personal competence and social competence.

Self-awareness and self-management scaffold an individual's personal competence, which involves controlling their individual emotions. Social awareness and relationship management constitute social competence, which is more concerned with how those emotions influence interactions with other people. Self-awareness involves "an ability to understand, make sense of, and perceive our emotions at any precise moment" (Pittaro, 2020). Self-awareness is the first of four essential skills for mastering emotional intelligence. Self-management, the second component of emotional intelligence, is the ability to manage emotional reactions to people and situations. Pittaro (2020) outlines that this involves more than resisting explosive or regrettable behaviour. He states that once an individual is aware of their emotional state, managing those emotions requires them to observe their breathing, assess what is likely to occur next, and evaluate how to quell a situation quickly and safely.

Social awareness is the third skill necessary for emotional intelligence and is an individual's "ability to accurately and quickly notice and assess the emotions of other people" (Pittaro, 2017b). They must understand the underlying reasons for other's behaviours and actions, such that they can respond accordingly. Relationship management is the final component of emotional intelligence. Bradberry and Greaves, cited in Pittaro (2020), explain that relationship management relies on abilities in the first three skills of self-awareness, self-management and social awareness. Relationship management focuses "on clear and concise communication skills and the effective handling of conflict or potential conflict" (Pittaro, 2017b) and is bound to social awareness.

In the context of corrections work, the applications of emotional intelligence are many. Pittaro (2020) provides examples such as where an offender tries to provoke a Correctional Officer by stepping into their personal space and screaming at them, they are most likely trying to evoke an emotional reaction. However, rather than giving them the reaction they want, it is important for the Correctional Officer to manage their emotions and respond in a calm manner.

Turner (2009) highlights that "officers with low emotional self-awareness may have a hard time acknowledging and verbalizing their own emotions and thus may experience difficulty in recognizing how their emotions affect interpersonal interactions, decision making, and overall functioning". Turner recognises that 
low self-awareness can cause a Correctional Officer to "avoid emotional ownership" and instead externalize what are really internal problems. Turner notes that this is a "situation that prevents them from asking for support when needed most". Low emotional self-awareness may further result in inaccurate projections of emotions and the misinterpretation of others' emotions. Judgments and decisions in these scenarios can become compromised. In the worst-case scenario, low emotional self-awareness can cause denial of personal feelings, thereby jeopardizing well-being. Such circumstances can cause an officer to overreact verbally or physically, causing embarrassment to themselves as well as to their departments.

By contrast, suggests Turner, "Officers with high emotional self-awareness are able to relate their own feelings to appropriate causes, thereby establishing good self-awareness". This positive attribute means Correctional Officers are able to differentiate between emotions and are able to understand how and why emotions change and they also have the ability to blend emotions appropriately. Officers with emotional self-awareness "have an understanding of how and why others affect them and are able to express these emotions and feelings in a positive way" (Turner, 2009). They know what they are feeling and why and can read other people and, similarly, they allow others to read them clearly. The officers are then more suitably equipped to prevent simple incidents from escalation and to "bring calm" to a potentially chaotic situation.

The competency tied to emotional intelligence that can have the greatest impact and meaning both within and outside the profession is empathy (Turner, 2009). Empathy is a term that defines an ability to sense other's emotions, coupled with the ability to appreciate what someone else might be thinking or feeling. Officers who demonstrate a high level of empathy can read other people's emotions and pick up on social cues. This, in turn, allows them to show concern for others. Turner posits that "officers lacking in empathy often fail to understand the feelings of others and have difficulties in relating to and with others, resulting in the misinterpretation of social cues and surprise reactions from others".

Stys \& Brown (2004) theorise that correctional service workers are amongst those who benefit most from a higher emotional intelligence. They outline that "their ability to read, understand, and react to inmate emotions as well as to manage their own emotions has a daily impact not only their own lives but on the lives of the inmates" (Stys \& Brown, 2004). They note that higher emotional intelligence could assist in the prevention of security incidents in an institution.

As an example of formalised application in emotional intelligence in corrections, the Building on Aboriginal Skills (BOAS) program can be considered. This is a culturally appropriate program that was designed for Aboriginal and Torres Strait Islander prisoners who want to reconnect with their land and culture while learning cognitive skills and positive behaviour (Australian Indigenous HealthInfoNet, 2020). The program is available in many regional prisons in Western Australia (WA) including Broome, Eastern Goldfields, Greenough, Roebourne 
and Casuarina prisons and is appropriate for both women and men. The program has been designed to utilise social learning theory encompassing "a learning approach that can support positive behaviour change, combining cognitive skills and emotional intelligence training" (Australian Indigenous HealthInfoNet, 2020). The program is characterised by key focus areas including life review, personal learning, self-management, social awareness and ongoing learning is delivered by prison officers.

\subsection{Emotional Intelligence and Health and Safety}

Ifelebuegu, Martins, Theophilus, \& Arewa (2019) state that "human error, negligence, carelessness and levels of intelligence affect the maintenance of stated standards of occupational health and safety during work routines". They also find that varying levels of emotional intelligence among workers are a major factor in determining workers' ability to uphold standards. Blondeel (2020) concurs noting that not recognizing negative emotions, or not knowing how to react to them, can result in: narrowing thinking; limiting interpretation of events; cause reactionary behaviour; cause demonstration of disengagement behaviours; reduce performance; cause employees to be more easily triggered; and, have lasting effects.

Pozniak (2017) considers the components of emotional intelligence with respect to their effects on health and safety. She outlines that "self-awareness has many directions of application such as bringing in subject matter experts on topics where you may not have expertise to recognizing situations within the workplace and addressing them with some creativity". The self-regulation aspect is key in being able to react to any condition or situation and seeing it as an opportunity. Pozniak believes this aspect gives individuals "an openness to change and the ability to find an approach that will work for everyone". Pozniak follows by highlighting that self-regulation "is the cornerstone that builds the perception that we are trustworthy and act with integrity which are seen as important components that allow us to influence others".

When an individual is emphatic, they see people's motivators or de-motivators for safety and see what they recognize as a hazard or how they assess the amount of risk. The emphatic individual can then look at things from the other's perspective and see the differences in perception. An individual can then talk "their language" and influence their view, definitions, motivation and desire and ability to act. Social skills stemming from emotional intelligence focus on the ability to find a common ground and build a rapport and using that rapport to move those needed to in the desired directions through influence and persuasiveness (Pozniak, 2017). Khandan \& Koohpaei (2016) posit that application of training courses for the development of components of emotional intelligence leads to the reduction of anxiety and stress and that can help prevent accidents and injuries in the workplaces. Improved emotional intelligence amongst Correctional Officers may, as such make a positive contribution toward maintaining health and safety in their work environment. 
In order to contribute to their respective work environments, effective application knowledge and skills in a workplace context may require development through learning which leads to a consideration of how these skills and knowledge can be taught.

\subsection{Teaching Emotional Intelligence}

A controversial aspect of emotional intelligence is whether or not it can be taught or developed (Stys \& Brown. 2004). Mattingly \& Kraiger (2018) demonstrated a moderate positive effect for training on development of emotional intelligence, regardless of the design of the training. However, their results suggest that trainees should acquire more emotional intelligence when they can discuss the meaning of the construct and how it applies to them, and they will learn less if they sit and listen.

Vohra (2013) in a search to understand what techniques are used by faculty to teach a course on emotional intelligence, found a reliance on case studies, role plays, stories (with a moral), discussions and write-ups related to EI abilities. Vohra also outlines that there are not many studies available to show which approach would be the best one in order to teach a course on Emotional Intelligence, and this is currently still the case.

Knowles (1970) suggests that adult learning is very different and distinct from child and proposed five assumptions in andragogy, the art and science of helping adults learn. The adult learner is self-directed and not dependent on others for direction. Vohra (2013) refers to Ozuah (2005), who confirms that "the learner has a need to know about educational training or development to aid them in self-directed goals so that optimum learning occurs". Adults enter the educational setting with more experience than children, and often incorporate their life experiences into their learning process. These learnings are shared with other learners, enabling the learners to learn from each other.

Adult Learning Australia (2020) outlines that Alexander Kapp and Eugen Rosenstock-Huessy developed theories around adult education, which were later popularised by Malcolm Knowles. Mowson (2018) further illuminates that in the 1970's, Malcolm Knowles coined the term "andragogy" referring to methods and principles used in adult education. Knowles (1970) used term "andragogy" to describe the art and science of helping adults to learn. In 1984, Knowles identified six adult learning principles including: The need to know; Self-concept; Experience; Readiness to learn; Orientation to learning; and Motivation.

Knowles (1990) outlines that "an andragogical approach is recommended as a way of enabling more meaningful outcomes for individuals". Within vocational education and training, there is evidence of an andragogical approach based on utilisation of flexible delivery, which supports the notion of learners as individuals in a specific or particular context and in the concept that learners are required to take responsibility for their own learning (Choy \& Delahaye, 2002).

The concept of andragogy then can emphasise the value of the process of learning. Watson (2015) elaborates that "it uses approaches to learning that are 
problem-based and collaborative rather than didactic, and also emphasises more equality between the teacher and learner". Trainers, on this basis, facilitate a learners' movement toward more self-directed and responsible learning as well as to foster the learner's internal motivation to learn and Adult Learning Australia (2020) outline that "motivation can be diminished by learning experiences that do not embrace adult learning principles".

Applying andragogical principles to technical education elicits any number of best practices in training provision. These include giving learners the opportunity to use their existing foundation of knowledge and experience gained from life experience and apply it to their new learning experiences (Watson, 2015).

To allow for a self-directed approach embracing adult learning principles, Vohra condones a Learning Conference (LC) technique for delivering training on emotional intelligence. This technique is selected as learners participate in setting their own learning personal agenda within a broader framework of group learning goals. This approach as creates a safe learning environment that enables experimentation and practice of new behaviours in place of older unchallenged and reactive behaviours. Experimentation and practice are most effective when they happen in conditions in which the learner feels safe. Further, learners acquire social competence in team work and learners can help each other.

The term Learning Conference in the context of self-directed Learning refers to the common consultation of all people involved in the learning process, stressing the seriousness and importance of common consultation for achieving self-directed learning (Vohra, 2013). The application of the Learning Conference was described by Kemper/Klein (1998), referred to in Klein, Reutter, Schelepa and Wenzig (2012), cited in Vohra (2013), and on the basis of four cornerstones: Individual Reflection; report round; interaction and group reflection; and, feedback to the moderator of the Learning Conference.

Vohra outlines that the four cornerstones offer a structured way to reflect on individual learning as a process and to take over responsibility by active participation and joint decision. In the individual reflection stage, the learners carry out self-reflective practices by themselves, with or without a framework provided by the facilitator. In the report round, the learners come together physically as a group to share what their experiences and learnings around the focus area have been. In the subsequent Interaction and group reflection round, the group as a whole discusses, clarifies, reflects, probes and crystallizes the data generated. In the last round, feedback to the moderator of the Learning Conference, the group concludes their discussion and sharing process, followed by a round of summarizing the key learnings of the group, conducted by the moderator. The learners assume responsibility for the moderation of the process and individual reflection is facilitated by the pre-work assessment sheets done by the learners.

The training structure presented by Vohra (2013) commences with introductory sessions aimed at informing learners about the various emotional intelligence models, definitions, latest research findings and the potential of the concept to increase one's effectiveness. The subsequent sessions introduce the 
Learning Conference technique in a structured way.

Learners are provided with self-report assessment sheets and worksheets to be completed before each session. Each worksheet deals with a specific emotional intelligence skill as its focus area and required in depth self-reflection and analysis on the part of the student in a structured way. A Learning Conference moderator is nominated for every LC and is provided with details on how to conduct a successful Learning Conference. Clear norms of participation, group work, commitment to pre-work, ownership around class learning are discussed and laid down.

The importance of creating a supportive group atmosphere is explained to learners and they are asked to maintain the confidentiality of the personal experiences shared by the learners. Each learner has to compulsorily participate in the discussions and this is the responsibility of the LC moderator. The class seating arrangement is a closed circular one, so that each student could see the others and have an opportunity to speak without any physical barrier between the student and the class. This also creates the image of a group working and discussing together.

The session is commenced by the LC moderator, with an introduction to the emotional intelligence skill in focus and its significance. The LC moderator then invites the group to volunteer and talk about their scores. Each section of the assessment sheet and the self-reflective worksheet is discussed by the entire group. The process relies on personal contribution and mindful listening. At the end of a discussion on every section of the worksheets, the moderator would summarise the key discussion points for the group. This is followed by inviting group members to speak on the exercise conducted and give feedback. The learners would be urged to implement learnings derived in the session and share feedback with the group in subsequent sessions.

Cherniss, Goleman, Emmerling, Cowan, \& Adler (1998) outline a process from planning to implementation for delivery of a training program in emotional intelligence. They present a flow chart suggests that there are four basic phases to the training process: Preparation for Change; Training; Transfer and Maintenance; and, Evaluating Change. Each of these phases is broken down into further sub-processes reflective of best practice in training and assessment.

Within the first phase, processes for preparation for change include an assessment of the organisation's needs on the basis that there are challenges that must be addressed related to both social and emotional training. Firstly, there will be those individuals who will be "skeptical about the link between emotional intelligence and the bottom line" (Cherniss et al., 1998). Secondly, to identify all of the particular competencies that are important for success. These should be addressed prior to commencing the training. The first phase also includes processes to assess personal strengths and limits, developing systems to provide feedback to participants, maximise learner choice and encouraging participation. During this initial stage the training should be linked to the learner's personal and learning goals. Expectations of learners are also ascertained at this point. 
During the second phase, Cherniss et al. (1998) outline that "in social and emotional learning, motivation continues to be an important issue during the training phase". They claim "the amount of time, effort, and potential threats to one's self-esteem that occur during social and emotional learning suggest that trainers continue to monitor the individual's motivation and intervene to bolster it". The training phase includes fostering a positive relationship between the trainer and learner, maximising self-directed change, setting clear goals, breaking goals into manageable steps, maximising opportunities to practice, and providing frequent feedback on practice. They outline that the training should be reliant on experimental methods such as role plays, group discussions, and simulations, that usually work better than lecturing or assigned reading for social and emotional learning. They do note, however, "even though experiential interventions seem to be especially productive for social and emotional learning, insight also can play a useful role. Insight serves as a natural link between situations, thoughts and feelings and enhances self-awareness, the cornerstone of emotional intelligence".

The third phase, transfer and maintenance of learned skills, identifies that "when learners return to their natural environments, there are likely to be many cues and reinforcers that support the old neural pathways that training was designed to weaken" (Cherniss et al., 1998). Cherniss et al. (1998) also acknowledge that "there may also be significant barriers to the use of some of the new social and emotional competencies that still have a fragile neural foundation". In order to reinforce the program, use of the newly acquired skills on the job must be encouraged. An organisational culture that supports learning is also essential for the reinforcement to take place. Cherniss et al. (1998) recognise that "the climate of the work environment is particularly important for transfer of social and emotional learning to the job".

The final phase requires evaluating change, usually through a process of conducting on-going evaluation and research. Through such evaluation, poor programs are improved and effective ones can be retained. Further, evaluation can be used to improve the training that is offered.

\subsection{Specifying Emotional Intelligence Competence}

In Australia, there is not a specific unit of competency related to emotional intelligence in corrections, limiting the opportunity for the currently popular micro-credentialing. At the time of writing, only one related unit is listed within the national register for training in Australia, being a Business Services Training Package (BSB) unit, BSBLDR511 Develop and use emotional intelligence. The BSB unit focuses on development of emotional intelligence in a workplace with regard to working with colleagues, team members and supervisors. As much as the unit covers development and use of emotional intelligence to increase self-awareness, self-management, social awareness and relationship management, it does not provide sufficient depth or context for implementation as a competency standard for a corrections worker. 
Correctional Officers are required to manage large groups of people with complex needs. There are no specifically related units within the CSC Correctional Services Training Package. The Corrections industry employs over 33,000 people across prisons, juvenile and immigration detention, parole services, correctional administration and management (Australian Industry Standards, 2019). The CSC Correctional Services Training Package defines competency standards for the industry.

Given that there are no specifically defined units of competency to cover the development of emotional intelligence in corrections workers, training is more likely to be delivered internally, or a workplace-based training programs until such time that suitable units are developed. Irrespective of delivery approach and methods, training should cover, at the least, the following performance criteria:

- Define and identify the benefits of Emotional Intelligence

- Define and practice self-management, self-awareness, self-regulation, self-motivation and empathy

- Identify and use evaluation criteria to determine own emotional strengths and weaknesses

- Identify personal stressors and own emotional states related to custodial settings

- Analyse and document potential emotional triggers in corrections work environments which may require determine appropriate emotional responses

- Evaluate the impact of own behaviours that demonstrate management of emotions

- Use self-reflection and feedback from others to improve development of own emotional intelligence

- Respond to the emotional states of offenders and assess emotional cues

- Identify and respond to offender trauma responses

- Develop a plan for identifying and responding appropriately to a range of cultural expressions of emotions

- Apply techniques to demonstrate flexibility and adaptability in dealing with offenders

- Demonstrate consideration of the emotions of offenders and colleagues when making decisions

- Create opportunities for others to express their thoughts and feelings in a non-threatening manner

- Assist offenders to understand the effect of their behaviour and emotions on others in the custodial setting

- Develop and implement plans to encourage the self-management of emotions in offenders and colleagues

- Develop and implement plans to encourage others to develop their own emotional intelligence, to build productive relationships, and maximise workplace outcomes

- Relate emotional intelligence to personal and professional leadership

- Encourage a positive, inclusive emotional climate in the custodial setting 


\section{Findings}

Learners should be able to demonstrate competence against a set of defined criteria at the completion of their training program. As outlined by Zeidner, Matthews, \& Roberts (2004) "emotional intelligence measures should be used in occupational contexts only if the instruments are specifically developed, normed, and validated to that end, and demonstrate adequate occupational relevance". Competency specifications within a national framework and specific to relevant industries, such as corrections, need to be developed and implemented such that the skills and knowledge can be effectively implemented. A range of training methods, such as Learning Conferences, can then be applied. Once these knowledge and skills are developed, emotional intelligence in a workplace context can make a positive contribution to a health and safety culture.

\section{Conclusion}

As identified by Australian Industry Standards (2019), the Corrections workforce needs to rely on individual cognitive skills and emotional intelligence to build rapport and sympathy with offenders in order to communicate effectively and resolve or de-escalate conflicts. Training programs in emotional intelligence are fundamental to facilitate Corrective Officers in undertaking their roles effectively as the corrections industry moves from an institutional to rehabilitative model. In order for the training programs to be effective, they should address a range of key competencies and should be delivered in a manner embracing adult learning principles.

\section{Conflicts of Interest}

The author declares no conflicts of interest regarding the publication of this paper.

\section{References}

Adult Learning Australia (2020). Adult Learning Principles. https://ala.asn.au/adult-learning/the-principles-of-adult-learning

Australian Indigenous HealthInfoNet (2020). Building on Aboriginal Skills (BOAS). https://healthinfonet.ecu.edu.au/key-resources/programs-and-projects/1233/?title=Buil ding\%20on\%20Aboriginal\%20Skills\%20\%28BOAS\%29\&contentid=1233_4

Australian Industry Standards (2019). Skills Forecast 2019: Corrections Industry Reference Committee.

https://www.australianindustrystandards.org.au/wp-content/uploads/2019/06/csc_sf20 19_final_pages_low_res.pdf

Basu, R. (2013). Emotional Intelligence of Correctional Officers of West Bengal. International Journal of Innovative Research and Development, 2, 220-227.

Blondeel, T. (2020). Does Emotional Intelligence Improve Safety? Results from One Large Manufacturer Bear Further Study. https://www.ehstoday.com/safety/article/21120248/does-emotional-intelligence-impro ve-safety 
Cherniss, C., Goleman, D., Emmerling, R., Cowan, K., \& Adler, M. (1998). Bringing Emotional Intelligence to the Workplace: A Technical Report Issued by the Consortium for Research on Emotional Intelligence in Organizations. The Consortium for Research on Emotional Intelligence in Organizations.

Choy, S., \& Delahaye, B. (2002). Andragogy in Vocational Education and Training: Learners' Perspective. https://www.avetra.org.au/data/Conference_2002_pres./2_Sarojni_Choy.pdf

Ifelebuegu, A. O., Martins, O. A., Theophilus, S. C., \& Arewa, O. A. (2019). The Role of Emotional Intelligence Factors in Workers' Occupational Health and Safety Performance-A Case Study of the Petroleum Industry. Safety, 5, 30. https://doi.org/10.3390/safety5020030

Kemper, M., \& Klein, R. (1988). Lernberatung: Gestaltung von Lernprozessen in der beruflichenWeiterbildung. Hohengehren.

Khandan, M., \& Koohpaei, A. (2016). Can Emotional Intelligence Be Used as a Tool to Control Occupational Accidents? Case Study in an Iranian Industry. Iranian Journal of Health, Safety \& Environment, 3, 406-412.

Klein, R., Reutter, G., Schelepa, S., \& Wenzig, A. (2012). The Conception, Councelling of Learning. http://www.learn-empoerment.org

Knowles, M. (1990). The Adult Learner: A Neglected Species (4th ed.). Houston, Texas: Gulf.

Knowles, M. S. (1970). The Modern Practice of Adult Education: Andragogy versus Pedagogy. Chicago, IL: Follett.

Mattingly, V., \& Kraiger, K. (2018). Can Emotional Intelligence Be Trained? A Meta-Analytical Investigation. Human Resource Management Review, 29, 140-155. https://doi.org/10.1016/j.hrmr.2018.03.002

Mowson, R. (2018). Adult Learning Principles and Styles: Area's to Consider When Delivering Training.

https://www.clearhorizon.com.au/adult-learning-principles-and-styles-areas-to-consid er-when-delivering-training/

Ozuah, P. (2005). First, There Was Pedagogy and then Came Andragogy. Einstein Journal of Biology and Medicine, 21, 83-87.

Pittaro, M. (2017a). How Emotional Intelligence Benefits Correctional Officers. https://inpublicsafety.com/2017/01/how-emotional-intelligence-benefits-correctional-o $\underline{\text { fficers }}$

Pittaro, M. (2017b). How Emotional Intelligence Benefits Correctional Officers: When Faced with a Volatile Situation, We Must Act with the Utmost Integrity and Ensure Our Actions Remain within the Legal and Departmental Boundaries of Our Jobs. https://www.correctionsone.com/corrections-training/articles/how-emotional-intellige nce-benefits-correctional-officers-6ZmCe3nVhX1CLFIF

Pittaro, M. (2020). Changing the Culture of Corrections: Transformational Leadership and Emotional Intelligence.

https://www.psychologytoday.com/au/blog/the-crime-and-justice-doctor/202001/chan ging-the-culture-corrections

Pozniak, E. (2017). Emotional Intelligence Important for Safety Professionals. https://www.thesafetymag.com/ca/news/opinion/emotional-intelligence-important-for -safety-professionals/187221

Queensland Government (2020). Custodial Correctional Officer. https://smartjobs.qld.gov.au/jobs/QLD-331181-19 
Salovey, P., \& Mayer, J. D. (1990). Emotional Intelligence. Imagination, Cognition and Personality, 9, 185-211. https://doi.org/10.2190/DUGG-P24E-52WK-6CDG

Stys, Y., \& Brown, S. L. (2004). A Review of the Emotional Intelligence Literature and Implications for Corrections. Research Branch, Correctional Service of Canada.

Turner, T. W. (2009). Understanding the Benefits of Emotional Intelligence for Officer Growth and Agency Budgets.

https://www.theiacp.org/sites/default/files/all/u-z/Understanding_the_Benefits_of_Em otional_Intelligence.pdf

Vohra, V. (2013). Self Directed Learning Approaches to Develop Emotional Intelligence in the Business School Context. International Journal of Human Resource Management and Research, 3, 47-56.

Watson, B. (2015). Adult Learning Theory and the Six Principles of Adult Learning. https://brucedwatson.wordpress.com/2015/05/05/adult-learning-theory-and-the-six-pr inciples-of-adult-learning

World Economic Forum (2018). The Future of Jobs Report 2018. Centre for the New Economy and Society.

http://www3.weforum.org/docs/WEF_Future_of_Jobs_2018.pdf

Zeidner, Z., Matthews, G., \& Roberts, R. D. (2004). Emotional Intelligence in the Workplace: A Critical Review. Applied Psychology: An International Review, 53, 371-399. https://doi.org/10.1111/j.1464-0597.2004.00176.x 